\title{
Approximation Models for Water Wave Equations
}

\author{
Leonard Bezati ${ }^{1}$, Shkelqim Hajrulla ${ }^{2}$ \& Kristofor Lapa ${ }^{2}$ \\ ${ }^{1,2}$ University of Vlora, Albania,
}

\begin{abstract}
:
In this work we are interested in developing approximate models for water waves equation. We present the derivation of the new equations uses approximation of the phase velocity that arises in the linear water wave theory. We treat the $(\mathrm{KdV})$ equation and similarly the $\mathrm{C}-\mathrm{H}$ equation. Both of them describe unidirectional shallow water waves equation.

At the same time, together with the (BBM) equation we propose, we provide the best approximation of the phase velocity for small wave numbers that can be obtained with second and third-order equations. We can extend the results of $[3,4]$. A comparison between the methods is mentioned in this article.
\end{abstract}

Key words: C-H equation, $\mathrm{KdV}$ equation, approximation, water wave equation, numerical methods.

\section{Introduction}

In many cases the numerical methods gives us a good approximation related to approximation of solutions. The finite-element method is a special case of the Galerkin method in which the base functions are chosen such that each base function becomes 1 at the corresponding nodes but otherwise 0 at other nodes. The Galerkin method permitted finite-element techniques to be extended into areas such as fluid mechanics, especially in water waves theory. Milder, Miles and Broer deal with the water waves on the Hamiltonian theory of surface waves (see [10], [11], [12]).

The KdV equation was originally derived by Korteweg and de Vries [7] from the model surface waves in a canal. The key to a soliton's behavior is a robust balance between the effects of dispersion and nonlinearity. Than we see the water wave equation with logarithmic term [1].

We are concerned with a two-dimensional, irrotational flow of an incompressible ideal fluid with a free surface under the gravitational field. The domain occupied by the fluid is bounded from below by a solid bottom. The upper surface is a free boundary and we take the influence of the gravitational field into account on the free surface. Our main interest is motion of the free surface, which is called a gravity wave.

The KdV equation was obtained by Benjamin [2]. We extend the Finite Volume Methods for Non-Linear Equations and results used in [3,4] and we assume that the waves are weakly nonlinear and weakly dispersive. In other words, the water depth is much smaller than the typical wavelength. Solitons are among the most interesting structures in nature. Being configurations of continuous fields, they retain their localized shape even after interactions and collisions.

The nonlinear surface water waves in perturbed problem are discussed by $[5,6]$ in the presence of the effect of surface tension. Based on the method of multiple-scale expansion for a small amplitude, they derived two $\mathrm{KdV}$ equations and discussed the two-soliton solution for $\mathrm{KdV}$ equations by using analytical methods. 
The main aim in this work is to effectively derive the KdV equations and employ the modified form of Laplace decomposition method introduced by Khuri [9] to establish approximate solutions of waves propagating along the interface between air-water.

The Galerkin method permitted finite-element techniques to be extended into areas such as fluid mechanics and series solution of some problems in elastic equilibrium of rods and plates (see [13]). Several other researchers have tested the validity of the KdV equation and variants in laboratory experiments (Remoissenet [9], Helfrich and Melville [8]). Their study includes a numerical scheme with error estimates, a convergence test of the computer code, a comparison between the predictions of the theoretical model and the results of laboratory experiments.

\section{Basic Equations}

The equation for the surface of water is $y=\eta(x, t)$ where $y=0$ represents the equilibrium situation. We assume that the motion is irrotational within the wave. Therefore, we can describe the wave inside the water by a velocity potential $\Phi(x, y, t)$ whose gradient is the velocity field:

$$
\left(\nabla \Phi=\frac{\partial \Phi}{\partial x}, \frac{\partial \Phi}{\partial y}\right)
$$

The divergence-free condition on the velocity field implies that the velocity potential $\Phi$ satisfies the Laplace's equation:

$$
\frac{\partial^{2} \Phi}{\partial x^{2}}+\frac{\partial^{2} \Phi}{\partial y^{2}}=0, \quad \text { for }-h<y<\eta(x, t)
$$

We consider the unsteady two-dimensional flow of inviscid, incompressible fluid in a constant gravitational field. The space coordinates are $(x, y)$ and the gravitational acceleration $g$ is in the negative $y$ direction. Let $h$ be the undisturbed depth of the fluid. The bottom of the fluid is assumed to have no topography at $y=-h$. This problem describes the interface dynamics, between air and water waves, under the gravity $g$.

Since the majority of shallow water flow of practical interest is two-dimensional, the governing differential equation can be written as:

$$
\frac{\partial^{2}(U u)}{\partial x^{2}}+\frac{\partial^{2}(U v)}{\partial x \partial y}+\frac{\partial^{2}(U \tau)}{\partial y^{2}}=\frac{p_{z}(x, y)}{S}+\frac{\partial(U h)}{\partial x^{2}}
$$

where, $p_{z}$ is the lateral pressure that is being applied, $S$ is the flexural deformation of the wave.

Here, $u$ and $v$ denote the velocity components in $x$ and $y$ directions, respectively, and $\tau$ denotes the depth of the free surface.

On a solid fixed boundary, the normal velocity of the fluid must vanish at $y=-h$

$$
\frac{\partial \Phi}{\partial y}=0 \quad \text { at } \quad y=-h
$$

which dictates that there is no flow perpendicular to the bottom. The boundary conditions at the free surface $y=\eta(x, t)$ are given by

$$
\frac{\partial \eta}{\partial \mathrm{t}}+\frac{\partial \eta}{\partial \mathrm{x}} \frac{\partial \Phi}{\partial \mathrm{x}}=\frac{\partial \Phi}{\partial \mathrm{y}}
$$


The Camassa-Holm $(\mathrm{CH})$ equation:

$$
\begin{gathered}
\left(u-u_{x x}\right)_{t}+3 u u_{x}=\varepsilon\left(2 u_{x} u_{x x}+u u_{x x x}\right), \quad x \in \Omega, \mathrm{t}>0 \\
u_{t}-u_{t x x}+3 u u_{x}-2 u_{x} u_{x x}-u u_{x x x}=0
\end{gathered}
$$

\section{Korteweg-de Vries Equations}

\subsection{Derivation of Korteweg-de Vries Equations}

In this section, we show that it is indeed possible to derive $\mathrm{KdV}$ equations [10] for the free surface $\eta(x, t)$ by using a multiple-scale perturbation theory. We introduce a small deformation parameter $\epsilon$. We get the system equations

$$
\begin{gathered}
\epsilon \frac{\partial^{2} \Phi}{\partial x^{2}}+\frac{\partial^{2} \Phi}{\partial y^{2}}=0, \quad \text { for } \quad-1<y<\epsilon \eta(x, t) \\
\frac{\partial \Phi}{\partial y}=0 \quad \text { at } \quad y=-1, \\
\epsilon \frac{\partial \eta}{\partial \mathrm{t}}+\epsilon^{2} \frac{\partial \eta}{\partial \mathrm{x}} \frac{\partial \Phi}{\partial \mathrm{x}}=\frac{\partial \Phi}{\partial \mathrm{y}} \quad \text { at } \quad y=\epsilon \eta
\end{gathered}
$$

To get a suitable expansion for the velocity potential $\Phi$, we note that from the Laplace equation (3.1) and the boundary condition (3.2), we may derive the following representation for $\Phi(x, y, t)$ as a series in $y+1$. Since $\partial \Phi / \partial y=0$ at $y=-1$,

$$
\Phi(x, y, t)=\sum_{n=0}^{\infty}(y+1)^{n} \Phi_{n}(x, t)
$$

\subsection{The Conditions Along the Boundaries}

Recall that the conditions on a simply supported edge parallel to the $\mathrm{y}$-axis at $x=a$, are.

$$
\begin{array}{r}
\left.U\right|_{X=a}=0 \\
\left.M_{X}\right|_{X=a}=-S\left(\frac{\partial^{2} w}{\partial x^{2}}+\vartheta \frac{\partial^{2} w}{\partial y^{2}}\right)_{X=a}=0
\end{array}
$$

On a simply supported edge parallel to the $\mathrm{x}$-axis at $y=a$, the change of $w$ with respect to the $\mathrm{x}$-coordinate vanishes; thus, the condition along this boundary are

$$
\begin{aligned}
\left.U\right|_{Y=a} & =0 \\
\left.M_{y}\right|_{Y=a}=-S\left(\frac{\partial^{2} w}{\partial y^{2}}+\vartheta \frac{\partial^{2} w}{\partial x^{2}}\right)_{Y=a} & =0
\end{aligned}
$$

The following part presents the numerical results achieved for the problems of approximation. All of the computation was carried out with the help of a symbolic algebra software system, Mathematica [16].

\section{Fundamentals of Modified Laplace Decomposition Method}

In this section, a brief outline of LDM is explained. For this, we consider the general nonlinear partial differential equation of first order (without loss of generality) in the following form: 


$$
L u(x, t)+R u(x, t)+N(u(x, t))=h(x, t)
$$

with the following initial condition:

$$
u(x, 0)=f(x)
$$

where $L$ is the first-order differential operator, $L=\partial / \partial t, R$ is linear differential operator, $N(u)$ presents the nonlinear term, and $h(x, t)$ is the source term. The methodology consists of applying Laplace transform first on both sides of (4.1)

$$
£[L u(x, t)]+£[R u(x, t)]+£[N(u(x, t))]=E[h(x, t)]
$$

Using the differentiation property of Laplace transform, we get

$$
s £[u(x, t)]-f(x)+£[R u(x, t)]+£[N(u(x, t))]=£[h(x, t)]
$$

Now, we will define the solution $(x$,$) by the series in the following form:$

$$
u(x, t)=\sum_{n=0}^{\infty} u_{n}(x, t)
$$

and the nonlinear operator $N(u)$ represented by an infinite series of the so-called Adomian's polynomials:

$$
N(u)=\sum_{n=0}^{\infty} A_{n}
$$

where $u_{n}(x, t), n \geq 0$ are the components of $u(x, t)$ that will be elegantly determined and $A_{n}$ are called Adomian's polynomials and defined by

$$
A_{n}=\frac{1}{n !}\left[\frac{d^{n}}{d \lambda^{n}} N\left(\sum_{i=0}^{\infty} \lambda^{i} u_{i}\right)\right]_{\lambda=0}, \quad n \geq 0
$$

Using (4.5) and (4.6) in (4.4), we get

$$
£\left\lfloor\sum_{n=0}^{\infty} u_{n}(x, t)\right\rfloor=\frac{1}{s} f(x) \frac{1}{s} £[h(x, t)]-\frac{1}{s} £\left\lfloor R \sum_{n=0}^{\infty} u_{n}(x, t)\right\rfloor+\frac{1}{s} £\left\lfloor\sum_{n=0}^{\infty} A_{n}\right\rfloor
$$

For numerical comparisons purpose, we construct the solution $(x$,$) such that$

$$
\lim _{n \rightarrow \infty} U_{n}(x, t)=u(x, t), \quad \text { where } U_{n}(x, t)=\sum_{i=0}^{n-1} u_{i}(x, t), \quad n \geq 0
$$

\section{Approximation of the Solution With a Polynomial}

The method has been used to solve problems in mechanical engineering such as structural mechanics, dynamics, fluid flow, heat and mass transfer, acoustics and other related fields. We consider the idea to approximate the solution with a polynomial involving a set of parameters. The polynomial is made to satisfy both the differential equation and the associated boundary conditions. Using orthogonality of polynomials, we can approximate the solution to the differential equation on shallow water wave equation.

Let's we have the second differential equation for a horizontal surface for water waves as follow 


$$
\frac{\partial^{2} U}{\partial x^{2}}+\frac{\partial^{2} U}{\partial x \partial y}+\frac{\partial^{2} U}{\partial y^{2}}=\frac{p_{z}(x, y)}{S}+\frac{\partial U}{\partial x^{2}}
$$

Consider a differential operator $L$, defined as

$$
L=\left[\frac{\partial^{2}}{\partial x^{2}}+\frac{\partial^{2}}{\partial x \partial y}+\frac{\partial^{2}}{\partial y^{2}}\right]
$$

So, eq. (2.1) becomes,

$$
L U=\frac{P_{z}}{S}
$$

We can express the solution to the above equation in terms of the eigenfunction and eigenvalues, which are defined as:

$$
L e_{n m}(x, y)=\lambda_{n m} L e_{n m}(x, y)
$$

where $e_{n m}(x, y)$ are the eigenfunctions and $\lambda_{n m}$ are the corresponding eigenvalues. Once the eigenfunctions and eigenvalues are known, it is possible to express $U(x, y)$ as,

$$
U(x, y)=\sum_{n=1}^{\infty} \sum_{m=1}^{\infty} u_{n m} e^{\lambda_{n m}} e_{n m}(x, y)
$$

Polynomial approximating functions will be used to represent the lateral deformation of the horizontal zone water. In this discussion, the trial function $e_{i}(x, y)$ will be represented as:

where

$$
\emptyset_{i}(x, y)=\sum_{i=1}^{N} \sum_{j=1}^{N} a[i, j] u_{j}(x, y)
$$

$$
u_{j}(x, y)=x^{L_{j}} \cdot y^{M_{j}}
$$

and, $L_{j}$ and $M_{j}$ are positive integers and $\emptyset_{i}(x, y)$ are coefficients to be determined.

\section{Conclusions and Discussion}

We see that the method mentioned in this article is one of the methods which were used to obtain the KdV equations. It is relatively short in mathematical calculation and more effective.

The Galerkin method can be used to approximate the solution to ordinary differential equations, partial differential equations and integral equations. The Galerkin method is used to determine the coefficients of these polynomials. The finite element and Galerkin methods are currently the standard numerical technique in use to solve various nonlinear problems.

In this study, we present model equations for surface water waves by using Laplace decomposition technique. It is used to estimate the KdV equations for the nonlinear theory, describing the behaviour of the perturbed system.

While the Hamiltonian expansions are complex in mathematical calculation. For the same, the DirichletNeumann operator expansions is relatively a long method when compared with the Galerkin method. 
Special attention is given to derive the solutions for the $\mathrm{KdV}$ equations, which describe the water waves propagation by using the MLDM, and then analyzed and discussed theoretically and computationally.

We observe that the elevation of the water waves is in form of traveling solitary waves, which increases in amplitude as the wave number increases.

Finally, the horizontal and vertical velocities have a nonlinear characters, which describe the physical situation of the system for free surface between air and water.

1. LDM does not require small parameters which are needed in perturbation method;

2. the method is very promising for solving wide application in nonlinear differential equations.

The extension of the general finite element method of structural analysis to water waves studies, since almost any particular situation can now be analyzed using a general purpose computer program.

In the last years, with the advent of the digital computer, other methods such as finite differences and finite elements have become practical. In 1956 Clough, Martin and Topp introduced the finite element method.

\section{References}

[1] Hajrulla, S.; Bezati, L.; Hoxha, F. Higher Order Wave Equation with Logarithmic Source Term. Preprints 2017, 2017120202 (doi: 10.20944/preprints201712.0202.v1).

[2] T. B. Benjamin, "Internal waves of finite amplitude and permanent form," The Journal of Fluid Mechanics, vol. 25, p. 241, 1966. View at Google Scholar

[3] D. J. Benney, "Long non-linear waves in fluid flows," Journal of Mathematical Physics, vol. 45, pp. 52-63, 1966. View at Google Scholar · View at Zentralblatt MATH

[4] Bezati, L., Hajrulla, S., \& Hoxha, F. (2018). Finite Volume Methods for Non-Linear Equations. International Journal of Scientific Research and Management, 6(02), M-2018.

[5] A. M. Abourabla, M. A. Mahmoud, and G. M. Khedr, "Korteweg-de Vries type equations for waves propagating along the interface between air-water," Canadian Journal of Physics, vol. 86, no. 12, pp. 1427-1435, 2008. View at Publisher · View at Google Scholar

[6] B. Deconinck, Solitons and Nonlinear Waves, Washington University, St. Louis, Mo, USA, 2007

[7] D. J. Korteweg and G. de Vries, "On the change form of long waves in a rectangular canal, and on a new type of long stationary waves," Philosophical Magazine, vol. 39, p. 422, 1895. View at Google Scholar

[8] S. A. Khuri, “A new approach to Bratu's problem," Applied Mathematics and Computation, vol. 147, no. 1, pp. 131-136, 2004. View at Publisher · View at Google Scholar · View at Zentralblatt MATH

[9] H. Liu and J. Yan, "A local discontinuous Galerkin method for the Korteweg-de Vries equation with boundary effect," Journal of Computational Physics, vol. 215, no. 1, pp. 197-218, 2006.

[10] Milder, D.M.1977. A note on: 'On Hamilton's principle for surface waves'.J. Fluid Mech.83(1), $159-161$.

[11] Miles, J.W.1977. On Hamilton's principle for surface waves.J. Fluid Mech.83(1), 153-158.

[12] Broer, L.J.F. 1974. On the Hamiltonian theory of surface waves. Appl. Sci. Res.29, 430-446.

[13] Galerkin, B.G (1915), Series solution of some problems in elastic equilibrium of rods and plates. Vestnik inzhenerov i tekhnikov 19(7), 897-908

[14] Remoissenet M (1999) Waves called solitons: Concepts and experiments, 3rd ed. Springer-Verlag, Berlin

[15] Helfrich KR, Melville WK (2006) Long nonlinear internal waves. Annu Rev Fluid Mech 38:395-425 
[16] Wolfram, S. (1989) Mathematica Addison-Wesley Publishing Company 